\title{
OTHONOMICS
}

Revista de economía, empresa y sociedad

Dossier «Realidades y desafíos de la Unión Europea»

NUEVOS RETOS EN LA UNIÓN EUROPEA

\section{La hoja de ruta hacia la creación de baronías energéticas europeas}

\section{Aurèlia Mañé Fstrada}

Universidad de Barcelona y Universidad de East Anglia

RESUMEN La hoja de ruta hacia una economía descarbonizada 2050 de la Unión Europea plantea que el objetivo de la política energética y medioambiental de la Unión Europea es conseguir reducir las emisiones de CO2, de cara al 2050, a un nivel inferior al 80\% del nivel de emisiones de 1990. Este artículo explicará, en su primer apartado, que este objetivo de descarbonización no significa apostar por una transición energética hacia las fuentes renovables, sino un cambio en el tipo y la localización de fuentes fósiles -«limpias»- utilizadas. En el segundo apartado se mostrará que las inversiones, infraestructuras y tratados que se proponen para llevar a cabo esta transformación conducirán a la creación de grandes monopolios energéticos y a la regionalización del espacio geoenergético europeo. Por último, concluiremos diciendo que si no se aplican medidas para compensar el poder de los monopolios, lo que crearán estas reformas serán unas baronías energéticas en el seno del espacio europeo.

PALABRAS CLAVE transición energética; Unión Europea; descarbonización; monopolios energéticos; política energética

\section{The roadmap for creating energy baronies}

\begin{abstract}
The Roadmap for Moving to a low-carbon economy in 2050 states that the objective of the European Union policy for Energy and Climate is to reduce its CO2 emissions to $80 \%$ below 1990 levels. First, the article explains that decarbonisation within this framework does not only mean a transition towards renewable energy, but also changes in the use of 'clean' fossil fuels, in type and location. Secondly, it shows that the investment, infrastructures and treaties proposed to carry out this transformation will lead to the creation of bigger monopolies, and to the regionalization of the European energy space. Finally, we conclude by saying that if measures to offset the power of the monopolies are not applied, the outcome of these reforms will be the creation of energy baronies.
\end{abstract}

KEYWORDS energy transition; European Union; low-carbon; energy monopolies; energy policy 


\section{Introducción}

En un momento como el presente, de cambio de modelo energético, cada vez es más difícil discernir qué propuestas tiene sobre la mesa la Unión Europea (UE) para hacer frente a este reto. A pesar de ello, una lectura entre líneas de la estrategia europea para la energía ${ }^{1}$ y el medio ambiente ${ }^{2}$, ambas bajo el paraguas de la Dirección General de Energía y Medio Ambiente, nos lleva a intuir que el objetivo de la política energética de la UE es, fundamentalmente, elaborar una normativa, la firma de unos tratados -en muchos casos bilaterales- y el desarrollo de una política tecnológica y de infraestructuras adaptadas al objetivo de la descarbonización competitiva. Objetivo que, de forma explícita, se establece en la hoja de ruta para el $2050^{3}$.

Históricamente, a ambos lados del muro de Berlín, la política energética fue el pilar sobre el que se fundó la construcción política europea. El núcleo originario de la UE fue la Comunidad Europea del Acero y del Carbón (CECA) y la Comunidad Europea de Energía Atómica (EUROATOM); mientras en el otro lado, en Europa del Este, uno de los símbolos de su integración en el Bloque Soviético fue su conexión al gasoducto Fraternidad (Druzhba), que unió la Unión Soviética con la República Democrática Alemana, atravesando Ucrania y los territorios de Europa Oriental. En las décadas de los cincuenta y sesenta, estos proyectos de infraestructuras tenían un claro significado político, el de creación de comunidades de paz, tanto en el este como en el oeste europeo. En la actualidad es difícil discernir el objetivo político de las políticas de la UE ampliada. A falta de definición, aunque suene extraño, el «modelo» energético europeo es un híbrido que se articula en torno a algunos proyectos de energías renovables, pero muy especialmente, en torno a la reestructuración "descarbonizada" de los viejos sectores del carbón, en Europa Central, de la energía nuclear y del gas proveniente del este. Esta opción otorgará un peso mucho mayor a la generación de electricidad, como principal fuente de energía secundaria; aunque su resultado será, más que crear una nueva Unión Europea, segmentarla, creando nuevas territorialidades.

\section{La descarbonización en la hoja de ruta para el 2050}

Desde que en el año 1997 se ratificara el Protocolo de Kyoto, que establece unos objetivos de reducción de las emisiones de $\mathrm{CO}_{2}$, causa del efecto invernadero y del cambio climático, se ha generado una confusión creciente entre los que deberían ser objetivos de las políticas energéticas y los que son objetivos de las políticas ambientales. Esta confusión se debe a que una de las principales causas de la emisión de $\mathrm{CO}_{2}$ a la atmósfera es la combustión de energía fósil (carbón, petróleo y gas). Por ello, con frecuencia en muchos ámbitos, también en la UE, se confunde transición energética con política ambiental.

Por este motivo, en la UE, desde 2009 se habla de un energy and climate package, que se va concretando en tres estrategias: el 2020 Climate and Energy Package ${ }^{4}$, el 2030 Framework for Climate and Energy Policies ${ }^{5}$ y el reciente Roadmap for Moving to a Low -carbon Economy in $2050^{6}$. A todo ello, hoy por hoy, independientemente de otras vías de financiación, está previsto dedicarle un 20\% del presupuesto de la UE, entre 2014 y 20207.

El objetivo del paquete 2020 era triple: a) que el 20\% de la energía generada en la UE tuviera como origen fuentes renovables, b) que se redujera en un $20 \%$ las emisiones de $\mathrm{CO}_{2}$ que van a la atmósfera y c) que aumentara la eficiencia energética en un $20 \%$. Hoy, ya con la hoja de ruta para el 2050, de lo que se habla es de energía

1. Esta estrategia se puede consultar en línea en http://ec.europa.eu/energy/index_en.htm.

2. Esta acción se puede consultar en línea en http://ec.europa.eu/clima/policies/package/index_en.htm.

3. Communication From The Commission To The European Parliament, The Council, The European Economic And Social Committee And The Committee Of The Regions, A Roadmap for moving to a competitive low carbon economy in 2050, COM (2011), 885 Final.

4. Véase la normativa y la documentación en http://ec.europa.eu/clima/policies/package/documentation_en.htm.

5. Véase la normativa y la documentación en http://ec.europa.eu/clima/policies/2030/documentation_en.htm.

6. Véase la normativa y la documentación en http://ec.europa.eu/clima/policies/roadmap/documentation_en.htm.

7. Sonja van Renssen (2012), New: the EU budget - it has never been so Green. http://www.europeanenergyreview.eu/site/pagina. php?id=3548 
y de sociedad hipocarbónica, y de una reducción de las emisiones de $\mathrm{CO}_{2}$ de un $80 \%$, en relación con el nivel que estas tuvieron en 1990.

Así, de la definición de este objetivo, se deriva un cambio en las formas de captura, transformación, generación y uso final de la energía. El resumen que de ello ofrece la Comisión Europea se puede ver en el gráfico 1.

\section{Gráfico 1. EU decarbonisation scenarios -2030 and 2050 range of fuel shares in primary energy consumption compared with 2005 outcome (\%)}
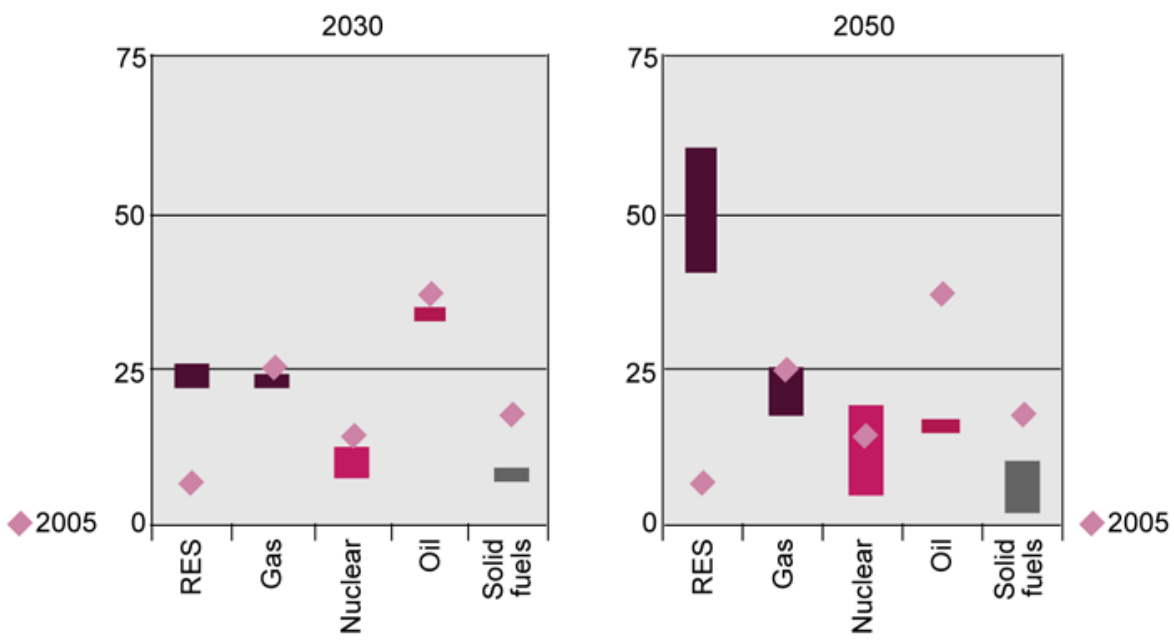

Fuente: Energy. Roadmap 2050. Unión Europea 2012.

Lo más relevante de estos datos es que el escenario futuro que plantean es un mix energético en el que, a la vez que se prevé un aumento en el uso de las fuentes de energía renovable, se mantiene el uso de las fuentes fósiles y de la energía nuclear. De hecho, solo hay un tipo de fuente en la que se vislumbra un descenso, y esta es el petróleo.

Una primera lectura de estos datos genera confusión, pues el escenario energético hipocarbónico que en ellos se plantea, para el 2050, apuesta por el carbón -camuflado bajo el término de combustibles sólidos. Carbón que, todavía, libera más $\mathrm{CO}_{2}$ a la atmósfera; además de seguir vaticinando un mix energético europeo con energía nuclear y gas. Así, esta primera lectura muestra que, a pesar del discurso dominante en el ámbito europeo de que la UE es líder en el uso de renovables, la realidad parece ir hacia otro lado. Por ello, es necesario entender qué nos están diciendo estos datos.

En primer lugar, nos informan de que cuando los responsables de la política energética de la UE hablan de una economía hipocarbónica, no tienen en mente un modelo energético basado en energías renovables. De hecho, lo que proponen es una cesta energética en la que, entre un 40 y un $60 \%$ de esta tendrá como origen fuentes energéticas finitas (energía fósil y uranio). Por esta razón, la estrategia energética de la UE se inscribe en la corriente dominante que define como energía limpia a aquella que, en el momento de generar electricidad o no libera $\mathrm{CO}_{2}$ -las energías renovales y la nuclear-, o emite menos $\mathrm{CO}_{2}$ que el petróleo -el caso del gas- o si lo emitiere, se cree que se podrá capturar, transportar y enterrar: el carbón.

En segundo lugar, los datos nos informan de la progresiva disminución del petróleo en la cesta energética europea. Esta es la transformación más significativa de esta propuesta. En pocas palabras, si se tiene en cuenta que la hoja de ruta para el 2050 no deja de abogar por las energías fósiles, el hecho de que se abandone el petróleo a favor del gas y del carbón significa:

a) que se está produciendo un cambio en la forma de generar energía, ya que se pasa de un modelo en el que predominan dos fuentes de energía secundaria (combustibles y electricidad) a uno eléctrico; pues poca electricidad se genera con petróleo y, mucha más, con carbón y gas, y 
b) que se está produciendo un cambio en la localización de las fuentes de energía primarias, pues se tiende a favorecer las autónomas -el petróleo y, tal vez, el gas y petróleo no convencional- y las cercanas, como el gas y, puede que, el carbón de los vecinos del este. Geoenergéticamente esta es una transformación muy significativa, ya que supondría un relativo desencaje de Europa occidental del bloque de los países consumidores de la OCDE y, por lo mismo, supondría una nueva subregionalización -con reminiscencias anteriores- del espacio energético europeo.

Por último, esta permuta de gas y carbón por petróleo, manteniendo la energía nuclear, apunta a que en la UE se apuesta por lo que se denominan energías de transición. Es decir, aquellas tecnologías que se considera que es posible implementar hoy en día, a la espera de la llegada del modelo energético alternativo, aunque realmente este tipo de tecnologías, por el tipo de inversiones e infraestructuras que llevan implícitas, condicionan el patrón energético existente para varias décadas ${ }^{8}$.

\section{Inversiones e infraestructuras derivadas de la hoja de ruta}

A la luz del objetivo de descarbonización por el que opta la UE y de la elección de fuentes de energía primara que se deriva de él, en la UE se definen varias líneas de actuación, que pivotan en torno a un eje central: la creación de un mercado -hipocarbónico- único de electricidad y de gas.

Además de la normativa requerida para crear este mercado y sus instituciones correspondientes, el grueso de las actuaciones previstas se centran en:

a) crear fondos y ayudas para que se invierta en eficiencia energética y tecnologías hipocarbónicas, con el fin de reducir las emisiones de $\mathrm{CO}_{2}$;

b) una significativa política de infraestructuras con el fin de crear una red eléctrica europea y corredores energéticos ${ }^{9}$, que comuniquen los lugares de extracción y generación de energía con los de su uso final, así como para crear sistemas de almacenamiento de energía ${ }^{10}$, dirigidos a flexibilizar el ajuste entre la oferta y la demanda de energía, y

c) un abanico de tratados bilaterales y regionales con y entre los territorios vecinos ricos en recursos naturales.

Concretamente ¿de qué estamos hablando?

Lo primero es que cuando la UE se refiere a tecnologías hipocarbónicas, se refiere a tres cuestiones muy concretas:

a) a la producción de electricidad por medio de las centrales - de gas- de ciclo combinado,

b) a la apuesta por las centrales térmicas de carbón de nueva generación, y/o

c) a la creación de infraestructuras de captura, transporte y almacenamiento de carbono, lo que se conoce con el acrónimo inglés CCS.

Originariamente, aunque después del trágico accidente en la planta Daiiichi en Fukushima las posibilidades ya no son tan claras, se contemplaba también una cuarta opción: la de las inversiones en fusión nuclear o en centrales nucleares de nueva generación. Por todo ello, la estrategia energética de la UE se dirige hacia la creación de grandes y centralizados núcleos de generación de energía, desde los que se transportará la electricidad a unos usuarios finales localizados en lugares alejados de estas unidades generadoras.

8. Véase Hermann Scheer (2011). El imperativo energético, págs. 94-123.

9. Véase http://ec.europa.eu/energy/infrastructure/strategy/2020_en.htm. Dos buenos resúmenes de estas estrategias son DG for Energy (2011), Priorities for 2020 and beyond - A Blueprint for an integrated European energy network y European Comission (2012), Connecting Europe. The energy infrastructure for tomorrow.

10. Véase el documento de la DG ENER Working Paper, The future role and challenges of Energy Storage a http://ec.europa.eu/ energy/infrastructure/doc/energy-storage/2013/energy_storage.pdf. 
En segundo lugar, la UE también hace mención a las renovables. La tipificación de este tipo de inversiones es menos claro, pues incluye dos tipos de opciones diferenciadas y, de facto, opuestas. Por un lado, se contemplan megaproyectos de generación de energía solar y eólica, que como los anteriores conducen a la creación de núcleos centralizados de generación eléctrica, alejados de los usuarios finales. Pero, por otro lado, también se contempla una panoplia de iniciativas descentralizadas de generación de energía renovable, con muchas reminiscencias con la energiewende alemana ${ }^{11}$. Así, lo que se plantea es un sistema de renovables dual.

Las opciones de generación condicionan la implantación de un sistema eléctrico de generación de electricidad en el seno del territorio europeo, que debería integrarse por medio de la creación de una red eléctrica europea -llamada European Network of Transmission System Operators for Electricity (ENTSO-E). Además, esta red debería tener la capacidad de transportar tanto la energía generada en el sistema descarbonizado (alta tensión y larga distancia), como la energía generada de manera descentralizada y renovable (baja tensión y corta distancia). También, con el fin de asegurar una mayor eficiencia y seguridad en el suministro energético, esta misma red se prevé inteligente (lo que llamamos smart grids), con el fin de trasvasar automáticamente los excesos de oferta que se den en un punto de red hacia el punto en el que se produzca un exceso de demanda, y viceversa; se prevé también que incorpore y esté conectada a sistemas de almacenamiento, que en la estrategia europea, más allá de los «tradicionales» embalses de agua, serán depósitos de gas.

El cuarto paquete de infraestructuras son las que se denominan corredores energéticos. Estos contemplan dos tipos de transporte: el de la energía primaria hacia el lugar de su transformación en energía útil (los de gas, petróleo e incluso se plantea del carbón) y el de la electricidad, las autopistas eléctricas. A día de hoy, como se observa en la tabla adjunta, la UE ha seleccionado doce proyectos prioritarios.

Tabla 1

\begin{tabular}{|c|c|c|c|c|}
\hline Priority corridors & $\begin{array}{c}\text { Total } \\
\text { investment } \\
\text { need (bn EUR) }\end{array}$ & $\begin{array}{l}\text { Estimated } \\
\text { Investment } \\
\text { gap (bn EUR) }\end{array}$ & $\begin{array}{l}\text { Average } \\
\text { co-financing } \\
\text { ratio need }\end{array}$ & $\begin{array}{l}\text { Likely need } \\
\text { for funding } \\
\text { (bn EUR) }\end{array}$ \\
\hline Northern Seas offshore grid & 30 & 8 & 0.10 & 0.80 \\
\hline $\begin{array}{l}\text { North-South electricity interconnections } \\
\text { in Western Europe }\end{array}$ & 30 & 5 & 0.10 & 0.50 \\
\hline $\begin{array}{l}\text { North-South electricity interconnections in } \\
\text { Central Eastern and South Eastern Europe }\end{array}$ & 40 & 12 & 0.20 & 2.40 \\
\hline BEMIP electricity & 5 & 3 & 0.50 & 1.50 \\
\hline $\begin{array}{l}\text { North-South gas interconnections } \\
\text { in Western Europe }\end{array}$ & 20 & 1 & 0.10 & 0.10 \\
\hline $\begin{array}{l}\text { North-South gas interconnections in Central } \\
\text { Eastern and South Eastern Europe }\end{array}$ & 26 & 5 & 0.20 & 1.00 \\
\hline Southern Gas Corridor & 22 & 8 & 0.10 & 0.80 \\
\hline BEMIP gas & 3 & 2 & 0.50 & 1.00 \\
\hline $\begin{array}{l}\text { Oil supply connections in Central Eastern } \\
\text { Europe }\end{array}$ & n.a. & n.a. & 0 & 0.00 \\
\hline \multicolumn{5}{|l|}{ Priority thematic areas } \\
\hline Smart grids deployment & 40 & 20 & & 1.00 \\
\hline Electricity highways & \multicolumn{4}{|c|}{ included in electricity corridors } \\
\hline $\begin{array}{l}\text { Cross-border } \mathrm{CO} 2 \text { network } \\
\text { (if technology viable) }\end{array}$ & 2.5 & 2 & & 0.02 \\
\hline TOTAL & 218.5 & 66 & & 9.12 \\
\hline
\end{tabular}

Fuente: Connecting Europe. The energy infrastructure for tomorrow. Comisión Europea.

11. Véase http://energytransition.de/. 
Estos doce proyectos configuran los siete clústeres regionales del mapa adjunto (gráfico 2). Estos, de facto, generan una parcelación del espacio energético europeo, pues muestran discontinuidades y solapamientos. Estas nuevas geografías de la energía en el seno de la Unión Europea, por el tipo de energía primaria empleada, tienen reminiscencias con los viejos espacios energéticos nucleares y carboníferos europeos, que ya existieron en el continente europeo; aunque debido al tipo de infraestructuras que se proponen llevarán a la creación de nuevos espacios geoenergéticos ${ }^{12}$.

\section{Gráfico 2. Priority corridors for electricity, gas and oil}

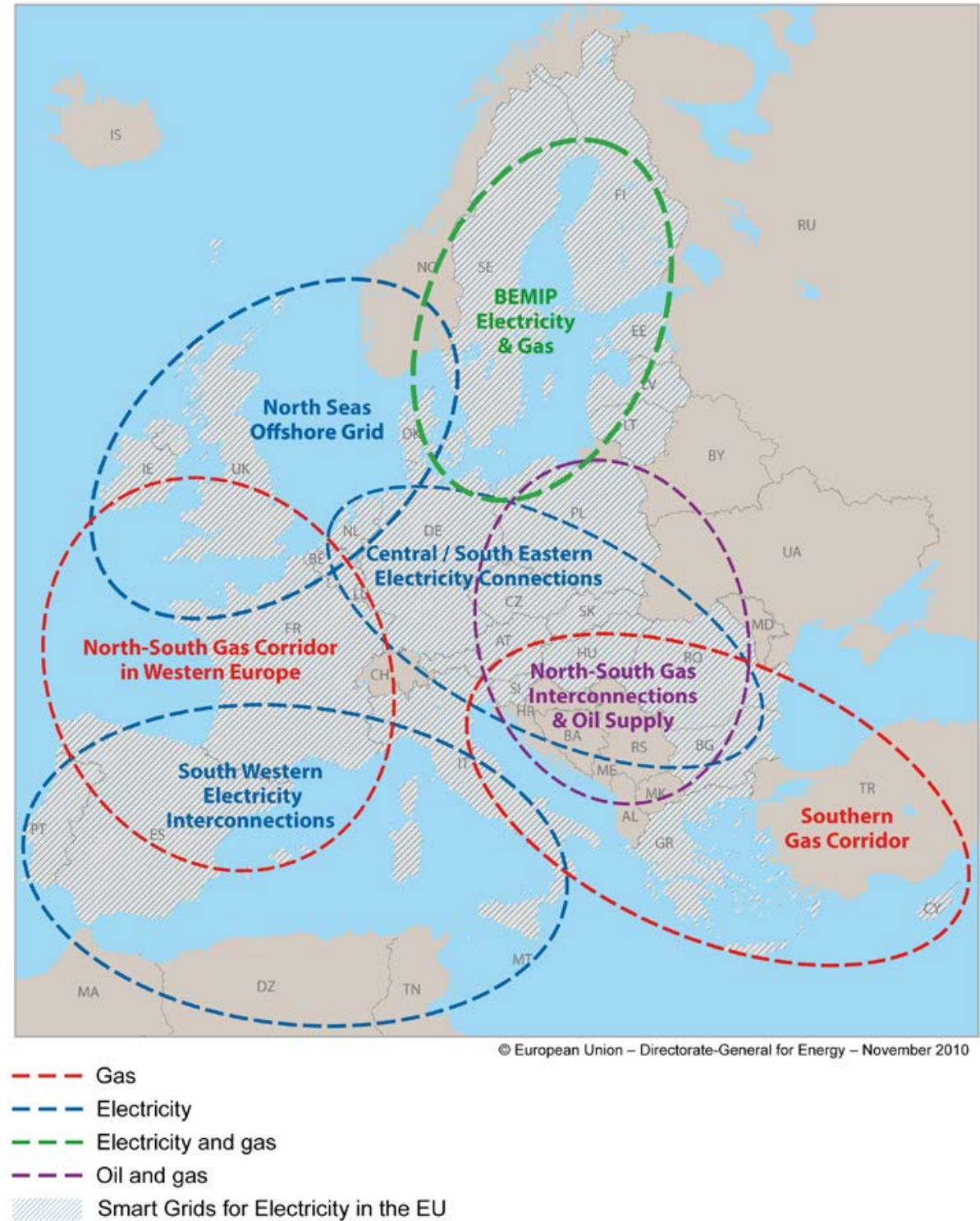

Fuente: Priorities for 2020 and beyond - A Blueprint foran integrated European energy network.

Unión Europea, 2011.

12. Un espacio geoenergético se define como una zona geográfica con una estructura de gobernanza energética. Para ser precisos, un espacio geográfico donde se institucionalizan un conjunto preciso de relaciones - de poder- energéticas entre los diferentes agentes activos en su seno: empresas energéticas y gobiernos de los territorios llamados productores y consumidores, territorios de tráfico... Este espacio suele ser más amplio que sus fronteras legales, ya que viene determinado por los agentes y los flujos energéticos. Véase Mañé-Estrada (2006). 
Estos cambios, necesariamente, se han de producir, debido a:

a) El cambio que suponen en las relaciones entre los lugares de consumo y de producción. Por ejemplo, en este escenario, Argelia, a pesar de ser una de las primeras productoras de gas mundial, pierde capacidad de influencia frente a los territorios cercanos al mar Caspio; o España, que es una de las principales potencias gasísticas de la UE y que incluso en algún momento se pensó que sería el lugar de tránsito privilegiado entre el Norte de África y Europa, pierde peso frente a los territorios del sudeste europeo.

b) Los cambios que se producirán en el seno de la industria, debido a la aparición de nuevas actividades -y empresas-, resultado de la opción hipocarbónica, con menos peso del petróleo.

c) Los cambios que se darán entre las empresas energéticas europeas. Siguiendo con el ejemplo del gas, empresas como Gas Natural ya han perdido relevancia frente a su socia francesa GDFSuez, aunque al mismo tiempo esta es un nodo secundario en relación con el operador noruego Gassco o en relación con lo que podrá ser el eslovaco Eurstream.

En definitiva, todo apunta hacia una nueva territorialidad de la energía en Europa. La territorialidad que se generará por el aumento en el uso de fuentes autóctonas (renovables y carbón) o cercanas, como el gas -además de la energía nuclear-, en detrimento de otras más alejadas de Europa, como el petróleo; y la territorialidad que se producirá por la construcción de un nuevo tipo de infraestructuras energéticas que conectarán localidades y empresas distintas. Todo ello creará una nueva red de relaciones de poder en las distintas regiones energéticas europeas (véase mapa 1).

\section{Conclusiones}

El somero análisis de las consecuencias derivadas de la implementación de la hoja de ruta para el 2050 hacia un sistema energético hipocarbónico realizado nos ha mostrado tres cuestiones fundamentales.

En primer lugar, que el mix energético hipocarbónico, a pesar de incluir una proporción de energía renovable, apuesta por el uso de energías fósiles -y nuclear- de carácter más europeo y plantea una cierta reducción en el uso del petróleo. Desde este punto de vista, la propuesta presenta ciertas reminiscencias con los proyectos político-energéticos a ambos lados del muro de Berlín, en las décadas de los cincuenta y sesenta, pero debido a su menor apuesta por el petróleo sugiere la emergencia de una nueva geopolítica energética europea.

En segundo lugar, debido al tipo de infraestructuras energéticas que propone, sobre todo destinadas a la creación de sistemas transeuropeos de producción de energía hipocarbónica, se priman las formas de generación de energía centralizadas, que además requieren grandes redes y corredores energéticos transfronterizos. Por ello, la estrategia apuesta por lo que Amory B. Lovins en su icónico artículo del año 1976 titulado «Energy Strategy: The Road Not Taken?» denomina hard energy paths, que son aquellos que conducen hacia estructuras integradas, verticales y monopolísticas de generación y distribución de energía. Estructuras que, a su vez, siguiendo las explicaciones de Lewis Mumford sobre los efectos políticos y sociales implícitos en las opciones tecnológicas, concluiremos que conducen a estructuras políticas y sociales autoritarias.

En tercer lugar, el tipo de infraestructuras propuestas apuesta por los acuerdos energéticos bilaterales con determinados vecinos e indica que se tenderá hacia la creación de clústeres regionales energéticos. Ello supone una modificación de las relaciones energéticas europeas, que se traducirá en un cambio en las relaciones de poder, políticas y económicas que gobiernan el sistema energético europeo, así como un cambio en la escala de estas, pues pasan de nacionales a regionales. En el presente estado de cosas, estas relaciones deberían ser gobernadas por los grandes monopolios energéticos europeos, pero es cierto que el que así sea dependerá de si existen otras fuerzas que puedan reequilibrar su poder. 
Desde este punto de vista, es necesario que la UE defina más claramente dos cuestiones adicionales. Primero, si más allá de la creación de un mercado gasístico y eléctrico europeo existe la voluntad de crear las instituciones necesarias para que el sistema energético europeo sea socialmente más justo que el que actualmente tenemos a escala nacional. Y, segundo, se deberá clarificar cómo se piensa gestionar políticamente un sistema que promueve, a la vez, la creación de islotes energéticos renovables, descentralizados y distribuidos, de tipo energiewende, y la creación de un sistema hipocarbónico centralizado. Hasta que no se tenga una respuesta clara a ambas cuestiones, es muy difícil valorar en qué acabara la hoja de ruta para el 2050 hacia una economía hipocarbónica. Dicho esto, y por ahora, todo apunta a que el resultado solamente será amplificar la escala de poder de los monopolios energéticos. Ello podría derivar en la creación de una especie de baronías energéticas en el seno del territorio europeo.

\section{Referencias bibliográficas}

EUROPEAN COMISSION (2012). «Connecting Europe.The energy infrastructure for tomorrow». [Paper en línea]. Disponible en: http://ec.europa.eu/energy/mff/facility/doc/2012/connecting-europe.pdf

EUROPEAN COMMISSION, DIRECTORATE-GENERAL FOR ENERGY (2011). «Priorities for 2020 and beyond - A Blueprint for an integrated European energy network». [Paper en línea]. Disponible en: http://ec.europa.eu/ energy/infrastructure/strategy/2020_en.htm

EUROPEAN COMMISSION, DIRECTORATE-GENERAL FOR ENERGY, Working Paper, «The future role and challenges of Energy Storage». [Paper en línea]. Disponible en: http://ec.europa.eu/energy/infrastructure/doc/ energy-storage/2013/energy_storage.pdf

EUROPEAN COMMISSION (2011). "Communication From The Commission To The European Parliament, The Council, The European Economic And Social Committee And The Committee Of The Regions, A Roadmap for moving to a competitive low carbon economy in 2050». COM (2011), 885 Final. [Comunicación en línea]. Disponible en: http://eur-lex.europa.eu/legal-content/EN/TXT/?uri=CELEX:52011DC0112

LOVINS, AMORY B. (1976). «Energy Strategy: The Road Not Taken?». Foreign Affairs. Núm. 65, octubre.

MAÑÉ-ESTRADA, A. (2006). «European energy security: Towards the creation of the geo-energy space». Energy Policy. Núm. 34, págs. 3773-3786.

MUMFORD, L. (2006). Técnica y Civilización. Madrid: Alianza Editorial.

SCHEER, H. (2011). El imperativo energético. Barcelona: Icaria\&Antrazyt. 


\section{Aurèlia Mañé Fstrada} amimanera@ub.edu

GATE (Universidad de Barcelona) \& Honorary Research Fellow, School of History (University of East Anglia).

De formación economista, con una posterior especialización en relaciones internacionales, es profesora de política económica internacional y de relaciones energéticas internacionales en la Universidad de Barcelona. Además de esta actividad principal, ha sido profesora asociada de la Joseph Korbel School of International Studies de la University of Denver (USA) y, en la actualidad, por sus trabajos en el ámbito de las transiciones y de la historia de la energía es honorary research fellow de la School of History de la University of East Anglia (UK).

Los textos publicados en esta revista están -si no se indica lo contrario- bajo una licencia Reconocimiento-Sin obras derivadas 3.0 España de Creative Commons. Puede copiarlos, distribuirlos y comunicarlos públicamente siempre que cite su autor y la revista y la institución que los publica (autoría, nombre de la revista, institución editora); no haga con ellos obras derivadas. La licencia completa se puede consultar en http://creativecommons.org/licenses/by-nd/3.0/es/deed.es.

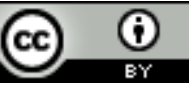

